Revue d'histoire de l'enfance « irrégulière »

Le Temps de l'histoire

$17 \mid 2015$

Naissance et mutation de la justice des mineurs

\title{
La loi canadienne sur les jeunes délinquants de 1908 : une loi sous influence américaine?
}

The Canadian juvenile delinquents Act of 1908: A law under American influence?

Jean Trépanier

\section{OpenEdition}

Journals

Édition électronique

URL : http://journals.openedition.org/rhei/3833

DOI : $10.4000 /$ rhei.3833

ISSN : $1777-540 \mathrm{X}$

Éditeur

Presses universitaires de Rennes

Édition imprimée

Date de publication : 30 octobre 2015

Pagination : 119-136

ISBN : 978-2-7535-4215-0

ISSN : 1287-2431

Référence électronique

Jean Trépanier, «La loi canadienne sur les jeunes délinquants de 1908 : une loi sous influence américaine ? », Revue d'histoire de l'enfance « irrégulière » [En ligne], 17 | 2015, mis en ligne le 30 octobre 2017, consulté le 30 avril 2019. URL : http://journals.openedition.org/rhei/3833 ; DOI : 10.4000/ rhei.3833 


\section{La loi canadienne sur les jeunes délinquants de 1908: une loi sous influence américaine?}

Le début du $x x^{\mathrm{e}}$ siècle est témoin de la naissance et du développement rapide de la justice des mineurs. Un modèle ressort de manière particulière : celui des tribunaux pour mineurs américains, qui incarnent ce que l'on a appelé le modèle protectionnel (ou, en anglais, le welfare model). Ce modèle est présent dans les débats de divers pays, même si leur influence varie d'un endroit à l'autre. Quelle influence ont-ils au Canada lorsque celui-ci adopte en 1908 une loi sur les jeunes délinquants qui régira les tribunaux pour mineurs canadiens pendant trois quarts de siècle? Les milieux de la protection de l'enfance canadiens en font la découverte en 1906 en participant à un congrès américain. Cette découverte sera à l'origine de la préparation d'un projet de loi que ces milieux tenteront de faire adopter par le parlement canadien, contre le gré du gouvernement, en recourant à l'aide de figures dominantes des child savers américains, et en prenant inspiration dans l'expérience américaine. La loi qui sera finalement adoptée porte nettement l'empreinte de cette inspiration.

The beginning of the $20^{\text {th }}$ century saw the birth and the quick development of juvenile courts. One model stands out: the American juvenile court, with its welfare orientation. It is part of the debates in various countries, even though its influence varies from one place to another. Did this model have any influence over the choices that were made in 1908 when the Canadian Parliament adopted the Juvenile Delinquents Act that was to govern juvenile courts in Canada for three quarters of a century? Children's aid societies discovered the American juvenile court in 1906 when taking part in a conference in the United States. This led to the preparation of a bill that these circles tried to push through Parliament, against the will of the government, with the help of leading figures of the American child saving movement, with the inspiration of the American experience. In the end, a bill was passed that bore clearly the imprint of this inspiration.

Mots-clefs: justice des mineurs, naissance et évolution, institutions pour mineurs, protection de l'enfance, naissance et évolution, justice et modèle protectionnel, délinquance des jeunes

Keywords : juvenile justice, birth and evolution, juvenile institutions, child protection, birth and evolution, justice and child welfare model, juvenile delinquency

\author{
Jean TrÉPANIER \\ Professeur émérite de \\ l'École de criminolo- \\ gie de l'université de \\ Montréal. Chercheur \\ au Centre internatio- \\ nal de criminologie \\ comparée (université \\ de Montréal) et au \\ Centre d'histoire des \\ régulations sociales \\ (université du Québec \\ à Montréal).
}


1. Sur l'affirmation de Kelso, voir HAGAN John et LEON Jeffrey, "Rediscovering delinquency: social history, political ideology and the sociology of law ", American

Sociological Review, 42/4, 1977, p. 587-598

Concernant l'Australie, voir Seymour, John, Dealing With Young Offenders, North Ride, N. S. W., The Law Book Co., 1988 p. 68-87. Pour la Norvège, voir NYQuIST Ola, Juvenile Justice, Londres, Macmillan, 1960, p. 139.

2. Loi du 22 juillet 1912 sur les tribunaux pour enfants et adolescents et sur la liberté surveillée. Le texte est disponible notamment sur le site suivant: [http:// www.criminocorpus.cnrs.fr/ lois/1945-174/media/uploads/ documents/loi1912.pdf]

3. Voir à ce sujet DupontBOUChat Marie-Sylvie, Pierre Éric, avec Fecteau Jean-Marie, Trépanier Jean, Petit Jacques-Guy,

SCHnAPER Bernard, DekKer Jeroen, avec la collaboration de Ménard Sylvie et Strimelle Véronique

Enfance et justice au xixe siècle. Essais d'histoire comparée de la protection de l'enfance, 1820-1914, France, Belgique, Pays-Bas,

Canada, Paris, Presses Universitaires de France, 2001. Concernant la France, voir en particulier aux pages 332 ss. Concernant

la Belgique, voir aux pages 354-355.

4. Loi concernant les jeunes délinquants, Statuts du Canada 1908, chapitre 40
T es Américains ont-ils été des pionniers en matière de justice des mineurs? Ce statut ne leur est guère contesté, ce qui n'empêche pas leur premier tribunal - celui de Chicago, fondé en 1899 - d'avoir eu des prédécesseurs. À titre d'exemples, l'Australie du Sud n'avait-elle pas établi un tel tribunal dix ans plus tôt, et les commissions de bien-être de l'enfance instituées en Norvège en 1896 n'en étaient-elles pas un équivalent administratif? Et que dire de l'affirmation du Canadien J. J. Kelso, alors surintendant des enfants négligés et dépendants de la province de l'Ontario à la fin du $\mathrm{XIX}^{\mathrm{e}}$ et au début du $\mathrm{XX}^{\mathrm{e}}$ siècle, à l'effet que Toronto avait été à l'origine des tribunaux pour mineurs, qu'il présentait comme une " initiative canadienne " que se seraient appropriés des " travailleurs sociaux américains ${ }^{1} »$ ?

Quelles que puissent être les prétentions d'acteurs autres que les Américains, il faut reconnaître que ces derniers conçurent un modèle auquel ils donnèrent une cohérence, lequel était issu d'une tradition juridique développée par les tribunaux américains au XIX ${ }^{\mathrm{e}}$ siècle sur la base de la doctrine parens patriae. Certaines expériences antérieures, comme celle de Toronto, se limitaient à la tenue de procédures visant les mineurs séparément de celles qui concernaient des adultes, sans y intégrer une orientation particulière aux mineurs sous la forme d'un modèle protectionnel ou l'équivalent. Ce dernier modèle, qui fut désigné en anglais sous l'appellation de welfare model, fut développé aux ÉtatsUnis, où il se répandit très rapidement dès le premier quart $\mathrm{du} \mathrm{xx}^{\mathrm{e}}$ siècle. Il en fut rapidement question à l'extérieur des États-Unis. À titre d'exemple, même si la loi française de 1912 sur les tribunaux pour enfants et adolescents et la liberté surveillée ${ }^{2}$ se tint à distance du modèle protectionnel, les débats qui précédèrent son adoption en firent état et certaines propositions débattues portèrent la marque des courants américains. De même, en Belgique, la loi de 1912 sur la protection de l'enfance s'inspira en partie des réalisations américaines après que le ministre de la Justice Carton de Wiart et son épouse aient participé au Congrès pénitentiaire international de Washington en 1910, en compagnie d'Adolphe Prins, et y aient puisé des idées qui modelèrent l'orientation à donner à la justice des mineurs dans leur pays ${ }^{3}$.

Le Canada est proche voisin des États-Unis, à la fois géographiquement et culturellement. Fit-il partie de ces pays qui puisèrent leur inspiration dans ce pays lors de l'adoption de sa loi sur les jeunes délinquants ${ }^{4}$ en 1908 ? La loi canadienne porte-t-elle la marque de l'influence américaine? La réponse à 
cette question passe certes par l'examen du contenu de la loi canadienne et sa comparaison avec l'orientation du modèle américain. Mais elle requiert aussi que l'on recourt à la micro-histoire pour voir et comprendre le rôle que jouèrent des Américains dans les événements qui menèrent à l'adoption de la loi. Nous procéderons donc en trois étapes. Nous situerons d'abord quelques éléments de contexte qu'il est essentiel de connaître pour comprendre le rôle et le statut de divers acteurs-clés. Nous verrons ensuite les événements qui, à compter de 1906, précipitèrent l'adoption de la loi - des événements où la présence américaine fut très réelle et, devrions-nous dire, utilisée. Nous poserons enfin la question de savoir si le contenu de la loi canadienne de 1908 porte la marque de l’influence américaine.

\section{QUELQUES ÉLÉMENTS DE CONTEXTE}

Deux éléments de contexte doivent en particulier attirer notre attention en raison du poids duquel ils pesèrent sur la suite des événements: les compétences attribuées par la constitution canadienne à divers ordres législatifs et gouvernementaux, ainsi que certaines lois adoptées à la fin du XIX ${ }^{\mathrm{e}}$ siècle.

\section{La constitution canadienne}

Depuis 1867 , le Canada est une fédération où les compétences législatives sont partagées entre un parlement fédéral et des législatures provinciales. Pour ce qui nous intéresse ici, le droit pénal et la procédure pénale relèvent de la compétence du parlement fédéral, peu importe qu'ils s'appliquent à des mineurs ou à des adultes. Par contre, la protection des enfants en danger est de compétence provinciale, tout comme l'est, pour l'essentiel, la mise sur pied et la gestion des tribunaux de première instance et des services pour les enfants en danger et les délinquants.

Donc, contrairement à la situation qui prévaut dans un grand nombre de pays, les lois relatives aux enfants en danger et celles qui visent les mineurs délinquants relèvent d'instances législatives distinctes, qui peuvent avoir chacune leurs propres visions des choses. On pourrait s'attendre à ce que le parlement fédéral - dont la responsabilité à l'endroit des mineurs délinquants tient à sa compétence en matière de droit pénal et de procédure pénale - adopte 
5. Acte concernant les écoles d'industrie, Statuts du Québec, 32 Vict. (1869), chapitre 17. Sur la genèse de ces écoles, voir notamment FECTEAU Jean-Marie, MÉnard Sylvie, Trépanier Jean et Strimelle Véronique, «Une politique de l'enfance délinquante et en danger: la mise en place des écoles de réforme et d'industrie au Québec (1840-1873) », Crime, Histoire et Sociétés / Crime, History and Societies, 2/1, 1998, p. 75-110.

6. OntaRIO, Report of the Commissioners Appointed to Enquire into the Prison and Reformatory System of Ontario, Toronto, Warwick and Sons, 1891 (Rapport Langmuir). La Commission est fréquemment désignée d'après le nom de son président, J. W. Langmuir, un ancien inspecteur provincial des prisons, asiles et institutions publiques de charité.

7. An Act for the prevention of cruelty to, and better protection of children, Statutes of Ontario, 1893, chapitre 45. une perspective éloignée des préoccupations de protection de l'enfance. Nous le verrons: tel ne fut pas le cas en 1908.

\section{Les lois antérieures}

Quelques lois adoptées dans la dernière décennie du XIX ${ }^{\mathrm{e}}$ siècle préparent le terrain en vue des événements qui mèneront à l'adoption de la loi de 1908. On parle ici de lois provinciales sur la protection des enfants en danger et de lois fédérales sur la tenue des procès visant les mineurs délinquants.

\section{Lois provinciales visant la protection des enfants en danger}

Retenant en cela le modèle britannique, la protection des enfants en danger au Canada passa tout d'abord par des placements en internat dans des écoles d'industrie, les écoles de réforme étant destinées aux mineurs délinquants. Au Québec, une loi sur les écoles d'industrie est adoptée en 1869 et l'ouverture de telles écoles se fait à compter de $1870^{5}$. En Ontario, le développement d'écoles d'industrie est suivi d'une première loi sur la protection de l'enfance, adoptée en 1888. Mais surtout, une commission créée par le gouvernement ontarien pour enquêter sur la situation des prisons et des écoles de réforme ontariennes - la commission Langmuir - remet son rapport en $1891^{6}$. S'appuyant sur une analyse des causes de la délinquance et sur une revue de pratiques ayant cours aussi bien en Ontario qu'aux États-Unis et en Europe, le rapport présente une série de recommandations dont certaines annoncent, elles aussi, la future loi sur les jeunes délinquants. On y recommande notamment que les procès des mineurs soient tenus non seulement à part de ceux des adultes, mais aussi en privé; que la détention des mineurs se fasse séparément de celle des adultes; que les placements en institutions soient ordonnés pour une période de temps indéterminée et qu'on puisse y mettre fin lorsqu'il est possible de retourner les mineurs dans la collectivité, en apprentissage ou autrement, au moyen de placements en familles d'accueil financés par l'État; que des organismes soient créés pour aider et surveiller les mineurs ainsi retournés dans la collectivité; que, de façon urgente, une loi soit adoptée pour mettre en place un système de liberté surveillée qui permette de prévenir des placements qui peuvent être évités.

C'est dans la foulée de ce rapport que, dès 1893 , le gouvernement ontarien fait adopter par la législature provinciale une nouvelle loi sur la protection de l'enfance $^{7}$. Reprenant l'orientation de la loi de 1888 , cette loi maintient une 
certaine association entre protection de l'enfance et prévention de la délinquance: si elle vise les enfants victimes de mauvais traitements, de négligence ou d'abandon, ceux qui sont orphelins de père et de mère ainsi que ceux qui sont privés de contrôle parental et d'éducation, elle vise également les enfants mendiants et vagabonds, ceux dont le parent survivant est emprisonné pour crime, tout comme ceux qui s'associent à des voleurs, ivrognes ou vagabonds ou qui fréquentent les prostituées. Elle réserve aux mineurs accusés d'avoir violé une loi provinciale des procès privés tenus séparément de ceux des adultes et précédés d'une évaluation de la situation du jeune. Elle favorise le placement en famille d'accueil plutôt qu'en institution. Des organismes devront être créés pour mettre ces politiques en œuvre. D'importants pouvoirs sont ainsi confiés à des comités visiteurs d'enfants et, surtout, à des sociétés d'aide à l'enfance, dont le rôle principal sera d'implanter ce recours à des mesures en milieu ouvert. Des sociétés d'aide à l'enfance seront ainsi implantées graduellement dans diverses villes ontariennes, incluant la ville d'Ottawa, ce qui sera important pour la suite des événements comme nous le verrons plus loin. Un «surintendant des enfants négligés et dépendants" sera nommé; ce haut-fonctionnaire sera le principal artisan de la mise en application de la loi. Le premier titulaire de ce poste sera J. J. Kelso ${ }^{8}$, un journaliste qui s'est illustré par ses campagnes et ses actions visant les enfants.

L'implantation de la loi connaît des débuts difficiles, ce qui n'empêche pas le développement considérable des interventions en milieu ouvert, grâce notamment aux sociétés d'aide à l'enfance. Une lacune devient toutefois de plus en plus évidente aux yeux de Kelso et de son entourage: ce type d'intervention devrait pouvoir toucher non seulement les enfants sur lesquels la loi provinciale permet une emprise (les enfants que l'on estime en danger), mais aussi ceux qui commettent des infractions aux lois fédérales (qui incluent le Code criminel), c'est-à-dire la majorité des mineurs délinquants. Cela, seule une loi fédérale pourrait le permettre vu les compétences exclusives du parlement fédéral en cette matière.

\section{Lois fédérales: le Code criminel et la loi de 1894}

L'année 1892 voit l'adoption du premier Code criminel canadien. Le code n'est pas muet en ce qui concerne les mineurs. Suite à des pressions de Kelso et d'autres Ontariens, le ministre fédéral de la Justice consent, avant son adoption par le Parlement, à y insérer une disposition permettant les procès séparés et
8. L'œurre de Kelso a été étudiée particulièrement par JONES Andrew et RUTMAN Leonard, In the Children's Aid: J. J. Kelso and Child Welfare in Ontario, Toronto, University of Toronto Press, 1981. 
9. Code criminel de 1892, article 550

10. Acte concernant l'arrestation, le procès et l'emprisonnement des jeunes délinquants, Statuts du Canada 1894, chapitre

11. L'application de cette partie de la loi de 1894 est limitée à l'Ontario en raison du manque d'infrastructures en matière de protection de la jeunesse dans les autres provinces.

12. L'usage de l'expression child savers pour désigner ces personnes qui, aux États-Unis, ont milité à l'époque en faveur du bien-être et de la protection de l'enfance nous a été tout particulièrement rappelé par PLATT Anthony M., The Child Savers: The Invention of Delinquency, $3^{e}$ éd., New Brunswick, Rutgers University Press, 2009. privés des mineurs de seize ans " autant que la chose paraîtra convenable et praticable $"$ ". Mais à l'expérience, le texte du code s'avérera trop peu contraignant à l'égard de magistrats peu enclins à trouver « convenable et praticable » la tenue de procès séparés et privés pour les mineurs. Suite à des campagnes menées par Kelso et d'autres, une nouvelle loi fédérale ${ }^{10}$ est adoptée en 1894 pour rendre obligatoire la tenue des procès des mineurs dans ces conditions, ainsi que pour permettre que, en Ontario ${ }^{11}$, un mineur délinquant puisse être confié à une société d'aide à l'enfance: un nouveau pas est ainsi franchi vers la création de tribunaux pour mineurs et vers l'engagement d'agences de protection de l'enfance dans le domaine de la délinquance des mineurs.

Bref, les développements qui marquent la fin du XIx ${ }^{\mathrm{e}}$ siècle touchent d'abord et avant tout la protection de l'enfance. Les sociétés d'aide à l'enfance ontariennes veulent favoriser l'intervention auprès d'enfants en danger dans leur milieu d'origine plutôt que sous forme de placements. De timides développements font que, dans une ville comme Toronto (la capitale de l'Ontario), le tribunal commence à entendre les affaires des mineurs délinquants séparément de celles des adultes - ce qui n'implique pas pour autant l'abandon de l'approche punitive traditionnelle.

Ce contexte facilitera l'adoption de la loi sur les jeunes délinquants. Mais certains événements déclencheurs devront survenir, où des Américains joueront un rôle important.

\section{L'ADOPTION DE LA LOI}

La narration des événements qui suit pourra sembler un peu anecdotique. Elle s'impose toutefois pour mettre en relief l'importance du rôle joué par certaines figures de proue du mouvement américain de ceux que l'on a appelés les child savers $^{12}$. La micro-histoire peut trouver ici son utilité.

Rappelons au départ qu'Ottawa est la capitale fédérale du Canada et qu'elle est située dans la province de l'Ontario. Elle fait donc partie de ces villes ontariennes qui, dans les années 1890 , se dotent d'une société d'aide à l'enfance. C'est l'année même de l'adoption de la loi ontarienne de 1893 que des notables de cette ville y établissent une société d'aide à l'enfance. William L. Scott fait partie du groupe. Juriste, il occupe une fonction dans l'administration judiciaire. Il est par ailleurs le fils du sénateur Richard W. Scott qui, en 1906, est secrétaire d'État dans le gouvernement de Wilfrid Laurier et leader du gouver- 
nement au Sénat. Les liens familiaux de William L. Scott allaient s'avérer essentiels pour faire adopter la loi sur les jeunes délinquants, à la fois en raison du rôle que jouerait son père, le sénateur Scott, et de l'accès aux cercles du pouvoir que lui conférait son appartenance à une famille de l'establishment de la capitale canadienne. En 1898, William L. Scott est élu président de la Société d'aide à l'enfance d'Ottawa; il le demeurera vingt-cinq ans et deviendra une des figures importantes du monde de la protection de l'enfance au Canada. Son rôle s'avérera déterminant pour l'adoption de la loi sur les jeunes délinquants ${ }^{13}$.

En mai 1906, se tient à Philadelphie la National Conference of Charities and Corrections. Comme d'autres organismes, la Société d'aide à l'enfance d'Ottawa est invitée à y envoyer des participants. À l'insistance des membres de son comité exécutif, Scott accepte d'y être délégué par la Société avec le secrétaire de celle-ci. Non sans réticence toutefois: il n'est nullement convaincu de l'utilité du déplacement. Comme il l'écrira plus tard:

Même si le nouveau tribunal des mineurs

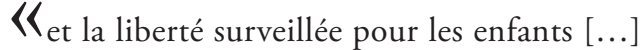
soulevaient déjà beaucoup d'intérêt chez les travailleurs sociaux des États-Unis, aucun d'entre nous à Ottawa n'en avions entendu parler et, je dois l'avouer, j'étais très sceptique que nous puissions tirer de cette conférence des idées nouvelles qui justifieraient les frais qu'impliquait ce voyage $e^{14}$.»

Une fois sur place, Scott change totalement d'idée. C'est l'occasion d'une véritable conversion:
13. Scott présentera plus tard une narration des événements ayant entouré l'adoption de cette loi: ScotT William L., The Genesis of the Juvenile Delinquents Act, manuscrit inédit, s. I., 1938. Nous avons largement puisé dans cette source d'informations utiles. Ce texte est en anglais; la traduction des passages que nous en citons est de nous. D'autres études ont aussi fait état de divers aspects des circonstances de l'adoption de la loi. Voir notamment DUPONT-BOUCHAT Marie-Sylvie et al., Enfance et justice..., p. 326-329, 343-347, 364-369; DuBols Pierre et TrÉPANIER Jean, «L'adoption de la Loi sur les jeunes délinquants de 1908: étude comparée des quotidiens montréalais et torontois ", Revue d'histoire de l'Amérique française, 52/3, 1999, p. 345-381; LEON Jeffrey S., " The development of Canadian juvenile justice: A background for reform ", Osgood Hall Law Journal, 15, 1977 p. 71-106; LEON Jeffrey S., "New and old themes in Canadian juvenile justice: The origins of delinquency legislation and the prospects for recognition of children's rights ", Interchange, 8 , 1977, p. 151-175; TRÉPANIER Jean et TuLKENs Françoise, Délinquance et protection de la jeunesse: aux sources des lois belge et canadienne sur l'enfance, Bruxelles, DeBoeck Université, 1995; TrÉPANIER Jean, « Juvenile courts after 100 years: past and present orientations », European Journal on Criminal Policy and Research, 7/3, 1999, p. 303-327; TrÉPANIER Jean, " Networks, lobbies and pressures: Children's aid societies, and the adoption of the Canadian Juvenile Delinquents Act of 1908 ", Paedagogica Historica, International Journal of the History of Education, 38/2-3, 2002, p. 581-605.

14. Scott William L., The Genesis..., op. cit., p. 7. 
15. /d., p. 7-8

16. Selon les indications fournies par Scott, The Genesis..., p. 8, il s'agirait d'un rapport préparé par Samuel J. Barrows, pour la Commission pénitentiaire internationale, intitulé

Children's courts in the United States; their origin, development, and results, publié en 1904 par le United States Government Printing Office.

17. Scott William L., The Genesis..., op. cit., p. 8.

18. Cela ne se produira pas qu'à Ottawa. À titre d'exemple, on procédera à Montréal à l'engagement d'une déléguée à la liberté surveillée en 1908, avant l'adoption de la loi sur les jeunes délinquants. Voir à ce sujet TréPANIER Jean, «Protéger pour prévenir la délinquance: l'émergence de la Loi sur les jeunes délinquants de 1908 et sa mise en application à Montréal ", Joyal Renée (dir.), Entre surveillance et compassion: l'évolution de la protection de l'enfance au Québec des origines à nos jours, Québec, Presses de l'Université du Québec, 2000, p. 49-95, à la p. 72.
Cette conférence nous apporta une véritable révélation. La question du 《tribunal des mineurs et de la liberté surveillée pour les enfants était dans les pensées de tous. Elle était mise de l'avant, exposé après exposé, dans tous ses différents aspects. [...] Nous rentrâmes à Ottawa pleins d'enthousiasme pour ce nouveau système ${ }^{15}$."

À Philadelphie, il noue des relations, tout particulièrement avec Madame Schoff, présidente du National Council of Mothers et l'une des responsables des opérations du tribunal des mineurs de Philadelphie. Il est spécialement impressionné par l'exposé de Madame Schoff sur la liberté surveillée comme on la pratique dans cette ville.

Scott rentre donc à Ottawa avec des perspectives nouvelles. Il rapporte aussi de la documentation pour les appuyer, incluant un document préparé par Madame Schoff sur la liberté surveillée et un autre publié par le gouvernement américain sur les tribunaux pour mineurs aux États-Unis ${ }^{16}$. Tribunaux pour mineurs et liberté surveillée sont intimement liés dans la perspective américaine: le délégué à la liberté surveillée est le bras droit du juge des mineurs. L'intérêt de Scott pour la liberté surveillée - partagé par d'autres personnes des sociétés d'aide à l'enfance ontariennes - se comprend bien : le travail que font les délégués auprès des mineurs délinquants correspond à celui que font les agents des sociétés d'aide à l'enfance auprès des mineurs en danger. Convaincus que leur travail fait merveille auprès des enfants en danger, les intervenants des sociétés d'aide à l'enfance ne voient que des bénéfices à l'étendre aux mineurs délinquants par le biais de la liberté surveillée.

Dès son retour à Ottawa, Scott obtient que deux dames soient engagées comme déléguées par la Société outaouaise, suivant ainsi l'avis de Madame Schoff selon lequel " tous les délégués à la liberté surveillée devraient être des femmes, même lorsqu'il s'agit de s'occuper de garçons ${ }^{17}$ ". Donnant suite à l'invitation que lui avait faite Madame Schoff, Scott envoie les deux nouvelles intervenantes à Philadelphie. Madame Schoff les y reçoit et leur dispense une formation de dix jours.

Le problème de l'absence de statut légal de la liberté surveillée demeure cependant entier: aucune loi fédérale n'assure la légalité de cette pratique. La pratique précède ici la loi ${ }^{18}$, mais il reste qu'une loi est nécessaire. À la mi-octobre 1906, Scott annonce à ses collaborateurs son intention de recom- 
mander au ministre fédéral de la Justice des ajustements législatifs pour corriger la situation.

Il se met lui-même au travail, disposant dans ses cartons des modèles desquels il peut s'inspirer. À la fin de novembre, il a rédigé un projet de loi sur les jeunes délinquants et deux projets de loi connexes ${ }^{19}$. Scott déclare lui-même que ce qu'il a préparé « suit de près les lois de l'Illinois et du Colorado [...] choisies par le Président Roosevelt dans son message au Congrès de décembre 1904 comme des modèles à suivre pour des lois de cet ordre, et qu'il incorpore aussi certaines caractéristiques intéressantes de la loi de la Pennsylvanie $e^{20} »$.

Il ne travaille pas en solitaire et consulte quelques personnes. L'une d'elles est son père, le sénateur ministre Richard W. Scott. Le sénateur Scott endosse l'avis de son fils: le gouvernement fédéral devrait présenter au Parlement un projet de loi visant l'institution de tribunaux pour mineurs et de la liberté surveillée, tels qu'ils existent aux États-Unis.

Il faut savoir que, à titre de secrétaire d'État, le sénateur Scott avait la responsabilité de préparer le discours du trône, où le gouvernement fait annoncer au parlement par le gouverneur général quels projets de loi il entend présenter au cours d'une session parlementaire qui commence. Cette préparation se fait en consultation avec chacun des autres ministres: ceux-ci l'informent des projets de loi qu'ils planifient de présenter au Parlement pour que le secrétaire d'État rédige à partir de l'ensemble un discours cohérent.

Un discours du trône doit être présenté le 22 novembre 1906. Le sénateur Scott y inclut que le gouvernement a l'intention de présenter un projet de loi sur les jeunes délinquants au cours de la session. Il oublie toutefois d'obtenir l'accord préalable de celui qui devrait présenter le projet au nom du gouvernement: le ministre de la Justice, Allen B. Aylesworth. Placé devant un fait accompli, ce dernier s'offusque et se braque, refusant de présenter un projet de loi dont, du reste, il semble rejeter l'orientation. Scott décrit ainsi la réaction qu'il a observée chez Aylesworth lors d'un échange qu'il a eu avec lui :

\footnotetext{
Il ne fit aucun secret de ce qu'il était sérieusement ennuyé et il refusa de se 《laisser apaiser. Pour un Libéral si robuste, son attitude à l'endroit des innovations était plutôt celle d'une personne très conservatrice et il est douteux que, peu importe les circonstances, il aurait appuyé le contenu de notre projet de loi. [...] Quant à l'omission de l'avoir consulté au préalable, il avait entièrement
}

\author{
19. Des deux projets de \\ loi connexes, l'un vise à \\ modifier une disposition \\ du Code criminel et l'autre \\ vise à établir des tribunaux \\ pour mineurs en Ontario, si \\ l'adoption d'une loi fédérale \\ sur les jeunes délinquants \\ habilite la province d'Ontario \\ à y procéder. \\ 20. Scotr William L., The \\ Genesis..., op. cit., p. 24
}


21. Id., p. 19-20

22. Le Canada fait alors partie de l'Empire britannique. Le gouverneur général représente le roi d'Angleterre auprès du gouvernement fédéral. Ses propos doivent être vus dans un contexte où, dans le régime britannique, le roi règne mais ne gouverne pas. Le même principe s'applique évidemment à son représentant tel que le gouverneur général dans un pays de l'empire. C'est au gouvernement et non au roi ou à son représentant qu'il revient de prendre des positions publiques sur les enjeux de politiques. Dans ce contexte, les propos de Lord Grey peuvent apparaître inattendus.

23. Scott William L., The Genesis..., op. cit., p. 22. 24. Id., p. 28 raison et, en conséquence, disposait d'une excuse excellente pour son hostilité à l'endroit de notre proposition ${ }^{21}$."

S'ensuivent des séries de manœuvres visant à faire pression sur Aylesworth pour l'amener à modifier sa position. On y fera appel à l'appui d'Américains éminents.

Scott invite tout d'abord Madame Schoff à Ottawa au début de décembre 1906. Une réception a lieu en son honneur dans les salles du président de la Chambre des communes. Suit un dîner auquel participent des invités de marque, dont le Premier ministre Laurier. Le lendemain, Madame Schoff prononce un discours public devant une salle comble. Quelques personnes y prennent la parole, dont le gouverneur général Grey, qui tient à remercier Madame Schoff et lui exprimer son accord avec les propos qu'elle a tenus ${ }^{22}$. Toutefois aucun membre du gouvernement ni du Parlement n'est présent.

Scott voit donc à organiser d'urgence une rencontre de Madame Schoff le lendemain à midi dans des locaux du Parlement. La rencontre est publicisée par écrit tôt le matin. À l'heure fixée, la salle est vide. À la demande de Scott, les présidents du Sénat et de la Chambre vont faire chercher les parlementaires et, après quelque attente, Madame Schoff peut s'adresser à une salle pleine de sénateurs et de députés qui l'accueillent chaleureusement. Aylesworth compte toutefois parmi les absents. Scott rapporte que le discours de Madame Schoff "fait une impression profonde ${ }^{23}$ ".

Les personnes présentes aux conférences de Madame Schoff ne sont pas seules à avoir connaissance de ses propos. Scott fait état de ce que nombre de journaux les rapportent et que de nombreux éditoriaux sont publiés à l'appui du projet de loi envisagé, notamment à Ottawa, à Montréal et à Toronto ${ }^{24}$.

Après le départ de Madame Schoff, Scott maintient la pression sur Aylesworth, lui demandant quelques minutes de son temps pour discuter du projet de loi. Il invoque s'être inspiré notamment des lois de l'Illinois, du Colorado et de la Pennsylvanie. Il évoque le témoignage du Président Roosevelt, qui a louangé ces lois comme des modèles à suivre. À cette occasion comme aux autres, la réaction d'Aylesworth est de nature dilatoire: il déclare ne pas disposer du temps nécessaire pour étudier la question comme elle le mérite.

Peu après, toujours en décembre 1906, Scott revient à la charge en transmettant à Aylesworth divers documents, incluant un rapport du gouvernement 
américain sur les tribunaux pour mineurs, la loi de l'Illinois ainsi que des articles portant sur le travail d'un célèbre juge pour mineurs américain, le juge Lindsey de Denver (Colorado).

En janvier 1907, un mois après le passage de Madame Schoff, Scott fait venir le juge Lindsey à Ottawa - un juge dont il écrit qu'à l'époque "son succès au tribunal des mineurs était la merveille du monde et que son nom était connu de tous ${ }^{25}$ ". Le 7 janvier, Lindsey prononce une conférence qui obtient encore plus de succès et fait l'objet de plus d'échos dans la presse que celle de $\mathrm{M}^{\mathrm{me}}$ Schoff. La grande salle est remplie de personnes d'Ottawa, de Montréal et de Toronto, incluant le gouverneur général, le chef de l'opposition à la Chambre des communes, des membres du gouvernement, des députés et des sénateurs. Mais en dépit de sa promesse, le ministre de la Justice est absent. Ce qui n’empêche pas le gouverneur général de proposer à l'assistance « un vote de remerciements au juge Lindsey pour son exposé si utile, ajoutant que le Canada et le monde lui devaient leur gratitude pour la lumière qu'il apporte sur ce qui a trait aux garçons ${ }^{26}$ ». Avec difficulté, Scott réussit à obtenir une rencontre privée d'une demi-heure entre Aylesworth et Lindsey. Il obtient même une rencontre pour Lindsey avec le Premier ministre Laurier - une rencontre où, selon Scott, Laurier aurait exprimé sa sympathie pour le mouvement et se serait exprimé dans des termes reconnaissant la nécessité de l'adoption d'une loi sur le sujet ${ }^{27}$.

En février, Scott fait parvenir de nouveaux documents au ministre de la Justice: un exposé de Homer Folks, président d'un comité de la législature de l'État de New York ayant étudié la question de la liberté surveillée, ainsi qu'un texte du juge Mack, le célèbre juge des mineurs de Chicago.

En dépit du succès public rencontré par les alliés américains qu'il a appelés à la rescousse, Scott réalise que le ministre de la Justice n'entend pas bouger. Une autre stratégie devra donc être utilisée. Comme le ministre de la Justice ne veut pas endosser le projet de loi et le présenter lui-même à la Chambre des communes au nom du gouvernement, le sénateur Scott le présentera au Sénat pour qu'il y soit débattu, sans pour autant mener son adoption à terme. L'objectif est d'en faire connaître publiquement les bienfaits, avec l'espoir que ce procédé suscitera des appuis divers qui convaincront le gouvernement d'endosser le projet et de le faire adopter par le parlement à une session ultérieure.

Le sénateur Scott présente donc le projet de loi au Sénat en avril 1907. Les sénateurs en débattent, ou plus précisément en vantent les mérites, lors

\author{
25. Id., p. 30. \\ 26. Id., p. 32. \\ 27. Id., p. 33.
}


28. Pour une analyse du contenu des journaux des deux principales villes canadiennes, Montréal et Toronto, voir DUBoIs Pierre et TrÉPANIER Jean, « L'adoption... ».

29. On peut le voir à divers indices, dont le fait que Béïque sera l'un des deux exécuteurs testamentaires de Laurier.

30. Scott William L., The Genesis..., op. cit., p. 84 de séances tenues en avril, après quoi la procédure parlementaire d'adoption est mise en suspens. Cette pause dans les procédures parlementaires ne signifie en rien la fin des activités, bien au contraire. On organise dans diverses villes canadiennes des pétitions publiques pour amener la population à manifester son appui au projet de loi. Un nombre considérable de personnes y apposent leur signature, particulièrement à Montréal où plus de cinq mille signatures sont recueillies. Des conférences publiques sont aussi tenues pour alimenter les débats. La presse en fait des comptes rendus, tout comme elle fait état des débats parlementaires ${ }^{28}$. Ces événements se tiennent en 1907 et 1908, alors qu'Aylesworth se montre toujours aussi inflexible.

Entre-temps, le sénateur montréalais Frédéric L. Béïque accepte de s'engager dans la défense du projet de loi. Juriste consommé, grand avocat lié aux milieux financiers, il a l'oreille et la confiance du chef du Parti libéral et Premier ministre Laurier $^{29}$. Béïque remanie considérablement le projet de loi, sans toutefois en modifier l'orientation.

À la session de 1908, Aylesworth résiste toujours. Alors que la fin de la session approche, accompagnée de rumeurs d'élections, les promoteurs décident de lui forcer la main en présentant la nouvelle version du projet de loi au Sénat pour l'y faire adopter, avec l'espoir que le ministre de la Justice se laissera fléchir une fois le processus législatif complété à la chambre haute. Le sénateur Béïque parraine le projet de loi et occupe l'avant-scène lors des débats sénatoriaux, qui ont lieu en mai et juin 1908.

Après l'adoption du projet par le Sénat, le ministre de la Justice accepte finalement de le présenter à la Chambre des communes au nom du gouvernement: il semble que le Premier ministre l'ait finalement exigé. Selon Scott, les pétitions (et tout spécialement celle de Montréal) auraient eu un impact déterminant sur cette décision ${ }^{30}$. Il est aussi plausible que, sachant qu'il allait déclencher des élections, Laurier ait voulu régler cet enjeu avant la fin de la session parlementaire pour éviter que ce débat ne vienne le hanter pendant la période électorale. Le projet de loi est rapidement adopté par la chambre basse, presque sans débats; deux amendements mineurs y sont apportés, auxquels le Sénat donne son accord. Le projet de loi est sanctionné le 20 juillet 1908.

Ce rappel des événements passe rapidement sur ce qui survint en 1908 . Non pas que cette année fut sans importance, bien au contraire: c'est l'année où, en fin de compte, tout se joua et où le projet de loi fut adopté. Il y a cependant 
moins à dire sur l'influence américaine: à cette étape, les stratégies choisies par les promoteurs du projet de loi ne font pas appel à leurs collègues américains. On fait bien sûr référence aux expériences américaines lors des débats parlementaires. On peut toutefois noter que les références à l'Angleterre y sont plus nombreuses. Ces évocations de ce qui se passait en Angleterre doivent cependant se comprendre dans le contexte où le Canada était à l'époque une colonie britannique: ce qui se passait au « Parlement impérial » faisait partie des références obligées et comptait parmi ces critères à l'aune desquels on jugeait de la valeur des projets soumis au Parlement canadien.

Cela dit, on ne se prive pas de faire référence à l'expérience américaine. Des figures emblématiques du pays voisin sont évoquées. On vise par là à mieux asseoir la crédibilité du projet. Du nombre, on compte des acteurs spécialistes du domaine alors fort connus : le juge Benjamin B. Lindsey de Denver ainsi que Madame Hannah Kent Schoff de Philadelphie, que les parlementaires avaient eu l'occasion de rencontrer à Ottawa; le juge Richard S. Tuthill de Chicago, premier juge du premier tribunal pour mineurs américain; le juge George W. Stubbs, d'Indianapolis, qui avait joué un rôle dans l'adoption de la loi de l'Indiana en 1903 et avait été le premier juge des mineurs de l'État ${ }^{31}$. On fait même référence à des discours prononcés par le Président Roosevelt ${ }^{32}$. On reconnaît et salue le rôle de pionniers joué par certains États américains et on souligne le fait que les innovations qu'ils ont apportées ont été rapidement adoptées par nombre d'autres États américains ${ }^{33}$. On présente les États-Unis comme faisant partie des pays représentant le progrès, au contraire du Canada que l'on déclare « en retard sur les autres nations progressistes ${ }^{34} »$. Le promoteur du projet de loi au Sénat en 1908 porte un jugement sur la qualité du projet de loi soumis à la Chambre haute en le comparant aux lois du Colorado, de la Pennsylvanie et d'autres États américains, établissant en quelque sorte celles-ci comme l'étalon en fonction duquel la comparaison peut être faite ${ }^{35}$. On évoque des exemples américains pour appuyer l'idée que le système n'est pas nouveau, qu'il a été mis à l'essai ailleurs et qu'on peut lui faire confiance ${ }^{36}$. On insiste sur la figure paternelle de ce juge tel qu'on le veut et tel que l'incarnent certains juges américains: le juge paternel, bienveillant, empreint de mansuétude et d'indulgence, qui cherche à établir un contact personnel avec l'enfant et ainsi gagner sa confiance, qui veut le sauver, et qui obtient les résultats recherchés ${ }^{37}$.
31. Sénateur Scotr, Débats du Sénat, 19 avril 1907, p. 880-882. Sénateur Elııs, Débats du Sénat, 24 avril 1907, p. 972. Sénateur COFFEY, Débats du Sénat, 21 mai 1908, p. 1057-1058

32. Sénateur Scotr, Débats du Sénat, 19 avril 1907 p. 881-882.

33. Sénateur Scott, Débats du Sénat, 19 avril 1907, p. 880. Sénateur Power, Débats du Sénat, 22 avril 1907, p. 904. Sénateur ElLIS, Débats du Sénat, 24 avril 1907, p. 972.

34. Sénateur LOUGHEEd, Débats du Sénat, 21 mai 1908, p. 1060

35. Sénateur BÉlQue, Débats du Sénat, 21 mai 1908, p. 1055

36. Sénateur Scotr, Débats du Sénat, 19 avril 1907, p. 880-881.

37. Sénateur Coffey, Débats du Sénat, 21 mai 1908, p. $1056-1058$. 
38. Cela est notamment confirmé par Scott, The Genesis..., op. cit., p. 83
En bref, que nous dit cette narration du processus d'adoption de la loi sur les jeunes délinquants pour ce qui a trait à la place qu’y tiennent les États-Unis? On peut y voir que, compte tenu de leur orientation et de leur expérience, les milieux canadiens de la protection de l'enfance étaient prêts à s'engager dans une démarche de mise en place de tribunaux pour mineurs et de services de liberté surveillée. Ils étaient désireux d'étendre aux mineurs délinquants une orientation qu'ils avaient mise en œuvre à l'intention d'enfants en danger. Il leur fallait cependant un élément déclencheur, des modèles et des appuis : les États-Unis les leur fournirent. Les réformateurs canadiens s'en saisirent et les utilisèrent, à la fois pour concevoir une formule qui serait adaptée au contexte canadien et pour vaincre les obstacles auxquels ils se heurtaient. Vu les oppositions rencontrées - tout particulièrement de la part du ministre responsable - on peut dire que le projet de loi fut adopté à l'initiative des milieux de protection de l'enfance; qu'il devint loi en dépit de l'opposition du gouvernement canadien; que le modèle américain offrit une source d'inspiration majeure; que certains acteurs américains jouèrent un rôle important, particulièrement lors des premières étapes de la préparation du projet de loi et des débats qui l'entourèrent; et que les références au modèle et aux réalisations des Américains firent partie de ces arguments d'autorité auxquels les parlementaires eurent recours pour établir la crédibilité du projet de loi lors des débats en chambre.

Cela dit, à quoi pouvons-nous voir les traces de l'influence américaine dans la loi elle-même?

\section{LA LOI ELLE-MÊME}

Le projet de loi présenté au Sénat par le sénateur Béique à la session de 1908 n'est pas identique à la version que le sénateur Scott avait proposée à la session de 1907. Le sénateur Béique ne s'était pas contenté de prendre le relais du sénateur Scott et de se limiter à défendre le projet en chambre: il avait retravaillé le projet de loi et se l'était approprié38. L’orientation était cependant restée la même. Il n'y a donc pas lieu d'examiner successivement les deux versions du projet de loi: un examen de la loi telle qu'elle fut adoptée convient tout à fait pour déterminer la cohérence avec le modèle américain.

Ce que l'on désigne comme modèle américain de l'époque est le plus souvent appelé welfare model ou, en français, modèle protectionnel. On comprend qu'il n’ait pas été appliqué de manière uniforme, même à l'intérieur des États-Unis, 
où chaque État a la compétence de légiférer comme bon lui semble en matière de protection de l'enfance et de réaction sociale à la délinquance des jeunes. Cela dit, il est des traits par lesquels on peut clairement l'identifier. Il se fonde sur la doctrine parens patriae, selon laquelle les affaires impliquant des mineurs ne doivent pas être vues comme des affaires pénales mais plutôt comme des affaires civiles, relevant du champ de la protection de l'enfance. La frontière entre affaires de délinquance et affaires de protection de l'enfance s'estompe. Les garanties protégeant les droits des accusés en matière pénale tombent: puisque les décisions sont vues comme étant prises dans l'intérêt du mineur, pourquoi lui reconnaitrait-on des droits qui lui serviraient à faire écarter des mesures dont il a besoin et qui lui semblent favorables? C'est ainsi que l'on opte pour une procédure informelle, qui vise à favoriser une communication simple et directe entre le juge paternel et l'enfant, fût-ce au détriment des protections que le droit pénal reconnaît normalement aux accusés.

Les mesures définies comme punitives n'ont plus leur place. Si l'objectif de la mesure n'est plus de punir, mais de protéger l'enfant pour qu'il ne devienne pas délinquant, le choix de la mesure doit être centré sur les besoins personnels du jeune, non sur l'infraction. Le principe de proportionnalité et les mesures à durée déterminée sont abandonnés. La mesure doit être appliquée aussi longtemps que le besoin s'en fait sentir. On peut la prolonger ou la modifier en cours d'exécution, selon l'évolution du jeune. Une fois le comportement délinquant ou la situation de danger établie devant le tribunal, le jeune peut demeurer pupille du tribunal ou de l'État jusqu'à sa majorité. Deux acteurs sont centraux pour la mise en ouvre du modèle. Tout d'abord, le juge des mineurs, paternel, bienveillant, protecteur, qui préside à une juridiction spécialisée, qui sait communiquer avec le jeune, qui veut le transformer, l'éduquer, non le punir. Il doit être choisi en raison de ses qualités humaines et de son aptitude à comprendre les enfants. Puis le délégué à la liberté surveillée, que les Américains et les Britanniques appellent l'agent de probation, qui est le bras droit du juge tant à la cour que dans la collectivité, qui intervient auprès du jeune et de sa famille en étant animé de la même perspective que celle qui est attendue du juge.

L'examen de la loi canadienne de 1908 montre qu'elle se situe tout à fait dans cette lignée. Si le préambule de la loi annonce que l'objectif fondamental qui est recherché est "le bien de la société ", le corps du texte de la loi indique bien que ce souci en côtoie un autre, qui n'est pas vu comme entrant en conflit 
39. Loi concernant les jeunes délinquants, paragraphe $16(5)$

40. Bibliothèque et Archives Canada, Documents de W. L. Scott, 27 octobre 1906 , cité par LEON Jeffrey, « New and old themes... », p. 154.

La traduction est de nous.

41. Aux États-Unis, la doctrine parens patriae fut utilisée pour définir les affaires de mineurs délinquants autrement que comme des affaires pénales, ce qui permit d'écarter pour les mineurs les protections légales prévues à la Constitution américaine en faveur des personnes accusées. À l'époque, la Constitution canadienne ne contenait pas une telle protection des droits des personnes accusées.

42. Loi concernant les jeunes délinquants, article 31.

43. Ce passage de l'article 31 de la loi canadienne est tiré mot pour mot de l'article 21 de la loi de l'Illinois. avec le premier: « La décision prise dans chaque cas doit être celle que la cour juge être pour le plus grand bien de l'enfant et dans l'intérêt de la sociétée ${ }^{39}$." William L. Scott explique le fondement de cette disposition:

il ne devrait pas y avoir de distinction claire et nette entre enfants négligés et $\ll$ enfants délinquants, mais tous devraient être reconnus comme faisant partie de la même classe, et devraient être traités en vue de servir les meilleurs intérêts de l'enfant ${ }^{40}$ ».

Techniquement, le Parlement canadien n’avait pas à invoquer la doctrine parens patriae pour assurer la constitutionnalité de sa loi, contrairement aux législatures américaines ${ }^{41}$. Il n'en retient pas moins l'esprit.

Il n'y a dès lors pas lieu de se surprendre qu'on y présente les jeunes délinquants non pas comme des criminels responsables d'actes qu'ils auraient délibérément décidé de commettre, mais plutôt comme des « enfant(s) mal dirigé(s), ayant besoin d'aide, d'encouragement et de secours ${ }^{42} »$. Leur responsabilité y est oubliée. L'accent est mis sur la nécessité de les protéger contre des facteurs qui les ont amenés à devenir délinquants, qu'ils soient d'origine familiale, sociale ou autre. On ne vise pas à sanctionner la commission d'une infraction, mais à adopter des mesures protectrices dont on attend qu'elles préviennent la récidive.

Cette préoccupation se traduit dans diverses dimensions du contenu de la loi. On y définit le principe qui doit guider les intervenants dans l'application de la loi : ils doivent adopter une approche semblable à celle des parents.

La présente loi doit être libéralement interprétée afin que son objet puisse être atteint, savoir: que le soin, la surveillance et la discipline d'un jeune délinquant ressemblent autant que possible à ceux qui lui seraient donnés par ses parents $[\ldots]$.

Il est frappant d'observer que, en rédigeant cet article central de la loi, les promoteurs du projet de loi recourent intégralement au texte qu’avaient adopté les législateurs de l'Illinois en $1899^{43}$. On réduit les garanties procédurales qu'offre normalement la procédure pénale: l'esprit dans lequel on veut aborder les procédures visant les mineurs délinquants les rend superflues, estime-t-on. On abandonne le principe de la proportionnalité: le choix de la mesure doit être fonction des besoins du mineur, non de la gravité de l'infraction qu'il a commise. Centrée sur le jeune plutôt que sur l'infraction, la mesure choisie pourra être modifiée en cours de mise en œuvre selon l'évolution du mineur 
et de sa situation; une nouvelle mesure pourra être imposée au jeune tant qu'il n'aura pas atteint l'âge de 21 ans, qu'il y ait récidive ou non. Une province pourra, si elle le juge souhaitable, faire en sorte que les affaires visant des jeunes déclarés coupables et pris en charge par certaines ressources soient transformées juridiquement en affaires de protection de l'enfance plutôt que d'être traitées en affaires de délinquance. On met en place des acteurs qui pourront traduire cette façon de voir dans leurs interventions: le juge de la cour des jeunes délinquants assisté du délégué à la liberté surveillée, dont les rôles sont calqués sur ceux que l'on a développés aux États-Unis. Ici encore, en définissant les fonctions du délégué à la liberté surveillée, la loi canadienne reprend mot pour mot les termes de la loi adoptée par l'Illinois en 1899. Le délégué est:

tenu de faire toute enquête que la cour exigera, d'être présent en cour afin de
représenter les intérêts de l'enfant lorsque la cause est entendue, de fournir à
la cour tous les renseignements et secours qu'elle juge nécessaires, et de prendre
soin de l'enfant avant ou après le procès, en la manière ordonnée par la courr ${ }^{44}$ ».

Aux yeux des promoteurs de la loi canadienne, le délégué correspond à l'intervenant de ces sociétés d'aide à l'enfance qui sont déjà à l'œuvre en Ontario pour protéger les enfants en danger.

Bref, exerçant à l'intention des mineurs sa compétence législative que la constitution lui reconnaît en matière de droit criminel et de procédure criminelle, le Parlement fédéral s'éloigne d'une perspective répressive et applique aux mineurs une approche de protection de l'enfance que l'on aurait pu croire l'apanage des provinces (auxquelles la Constitution confie la compétence en matière de protection de l'enfance). Au-delà des obstacles constitutionnels - et malgré eux - il endosse des orientations semblables à celles que choisissent divers pays, dont tout particulièrement les États-Unis. Ceux-ci avaient eu recours à la doctrine parens patriae pour contourner les obstacles que dressait leur Constitution. Le Parlement canadien procède différemment pour atteindre les mêmes objectifs, en donnant à la loi pénale visant les mineurs un visage de protection de l'enfance. Tout en ayant ses particularités, la loi canadienne de 1908 endosse les orientations qui ont été développées aux États-Unis, retenant même à l'occasion des passages intégraux de textes qui y ont été adoptés.
44. Cet extrait de l'article 27 de la loi canadienne reprend textuellement la partie de l'article 6 de la loi de l'Illinois qui définit les fonctions du délégué. 


\section{ConClusion}

Les premières années $\mathrm{du} \mathrm{Xx}^{\mathrm{e}}$ siècle demeurent un moment marquant pour la justice des mineurs. C'est le temps de ses débuts. C'est aussi celui de grands débats et des choix sur l'orientation qu'elle doit prendre. Les États-Unis y jouent un rôle central. Ils développent un modèle qui, pour certains, devrait servir d'inspiration et, pour d'autres, ne pas être retenu. Pensons à titre d'exemple que deux pays voisins, la Belgique et la France, adoptent tous deux en 1912 des lois qui orienteront pendant quelques décennies leur justice des mineurs respective, la première optant pour le modèle protectionnel inspiré des Américains, mais non la seconde.

Pour ce qui concerne le Canada, les pages qui précèdent montrent clairement que non seulement ce pays décide de suivre le courant proposé par les Américains, mais qu'il le fait à la suite d'influences venant des États-Unis. En se rendant à Philadelphie en mai 1906, William L. Scott ne se doutait pas de ce que sa participation à un congrès enclencherait. Il n'avait pas prévu que ce qu'il y trouverait l'amènerait à jouer un rôle central dans la préparation de la loi qui régirait la mise en place et la consolidation de la justice des mineurs au Canada pendant trois quarts de siècle. Cela fut possible parce qu'il trouva à Philadelphie et ailleurs aux États-Unis des perspectives et des alliés dont les orientations convergeaient avec les siennes. La création des sociétés d'aide à l'enfance ontariennes avait préparé le terrain. C'est ce qui permit à l'influence américaine de jouer. Cette influence prit au départ la forme d'une séduction: Scott et ses collègues furent conquis par les perspectives qu'ils avaient découvertes aux États-Unis. Elle devint ensuite plus active, fournissant des documents utiles à la préparation d'un projet de loi et apportant une contribution pour vaincre des obstacles par la suite. L'influence américaine fut très présente dans ce contexte. Tout comme elle le sera 90 ans plus tard lorsque le Parlement canadien optera pour une tout autre orientation. Mais cette histoire récente sera à faire ailleurs. 